\title{
CONTRIBUIÇÃO À HERPETOLOGIA DA ARGENTINA E DO RIO GRANDE DO SUL, BRASIL POR WILLIAM WRIGHT MILSTEAD (AMPHIBIA, REPTILIA)
}

\author{
Thales de Lema ${ }^{1}$ \\ Pedro Canísio Braun ${ }^{2}$
}

\begin{abstract}
CONTRIBUTION TO THE HERPETOLOGY OF ARGENTINA AND RIO GRANDE DO SUL, BRAZIL BY WILLIAM WRIGHT MILSTEAD (AMPHIBIA, REPTILIA). In this paper we are presenting the list of the specimens and the taxa obtained; there are 73 names of species belonging to 40 genera, of which 32 species and 13 genera are amphibians, and 42 species and 27 genera are reptiles. There are several field notes of Milstead and many data on the snakes studied by Lema with Milstead. The collection was widespread by Milstead to several institutions, but mainly to Field Museum of Natural History, Chicago, and Museu Nacional do Rio de Janeiro, Brazil.

KEY WORDS. Zoogeography, Amphibia, Reptilia, Argentina, Brazil
\end{abstract}

Esta publicação foi motivada pelo desejo de homenagear o Dr. William Wrightt Milstead, herpetólogo ecologista que esteve residindo em Porto Alegre durante três anos, tendo se dedicado ao colecionamento de exemplares da herpetofauna da área. Sua dedicação despertou o interesse de professores e alunos da Universidade Federal do Rio Grande do Sul, que passaram a colecionar espontaneamente, tendo deixado amigos e uma lacuna com seu falecimento posterior.

Milstead veio ao Brasil procedente dos Estados Unidos da América, como ecologista, para trabalhar em projeto do Departamento de Genética da Universidade Federal do Rio Grande do Sul. Sua vinda decorreu do pedido feito pelo chefe do departamento, Dr. Antonio Rodrigues Cordeiro, à Rockeffeler Foundation, financiadora do projeto, e que tratava do estudo de populações naturais de Drosophilidae. Mas Milstead era herpetólogo e, uma vez em Porto Alegre, dedicou-se totalmente ao colecionamento herpetológico.

Milstead saía com as viaturas do então Instituto de Ciências Naturais (hoje repartido em Instituto de Geociências e Instituto de Biociências). Coletava, geralmente, nas rodovias. Com o tempo, professores e alunos aderiram, auxiliando consideravelmente a coleção. Para o tombamento dos especimens e de dados, organizou diversos fichários, cujas fichas tinham cores diferentes

1) Instituto de Biociências, Pontifícia Universidade Católica do Rio Grande do Sul, Caixa Postal 1429, 90619-900 Porto Alegre, Rio Grande do Sul, Brasil. Bolsista do CNPq.

2) Fundação Zoobotânica do Rio Grande do Sul, Caixa Postal 1188, 90001-970 Porto Alegre, Rio Grande do Sul, Brasil. 
indicando diversos tipos de dados como, por exemplo, alimentação, habitat, abrigo, etc.

Durante sua estada, ministrou cursos de sistemática e ecologia de anfíbios e répteis, a nível de extensão, que foram cursados pelo autor sênior travando conhecimento e passou a auxiliar em seu trabalho de campo e de laboratório.

Permaneceu no Brasil de outubro de 1953 a junho de 1955, tendo ultrapassado seu prazo de afastamento e perdendo seu emprego (Ross State College, Alpine, Texas). Soubemos que esteve trabalhando, antes de falecer, na Universidade de Boulder, Colorado.

$\mathrm{O}$ autor sênior doou a Milstead uma pequena coleção de anfíbios e répteis, exceto serpentes (com as quais trabalhava), e que aumentaram a amostragem herpetofaunística da região do planalto oriental da região.

Outros professores do Instituto que auxiliaram Milstead foram, Dr. Jorge Alberto Petersen (falecido) e Dr. Celso Paulo Jaeger, sendo Jaeger o auxiliar de direção do Departamento de Zoologia na ausência do Dr. Jorge Godofredo Felizardo (Titular). O próprio Prof. Cordeiro participou de viagens de captura, entrando à noite nos banhados.

A coleção organizada foi, pouco antes da partida de Milstead, distribuída por ele a várias instituições (vide Material e Métodos). Os exemplares que ficaram para o Departamento de Zoologia foram incorporados pelo autor sênior à coleção existente e que fora organizada por Oscar Miranda Fróes. Esta coleção foi, posteriormente, reorganizada pelo laboratorista Sívio Mendes Correa, ocasião em que foi adicionada à mesma a coleção particular de Lema. Posteriormente, Lema reassumiu a coleção, que estava abandonada, mas não teve condições de levá-la a bom termo.

Milstead participou de uma viagem à Argentina, organizada pelo Departamento de Genética, sendo auxiliado na coleta pelo Prof. Cordeiro, durante os meses de janeiro a fevereiro de 1954. O trajeto foi de Porto Alegre a Uruguaiana e daí à Bahía Blanca, na Argentina, retornando aproximadamente igual (Fig. 1). Os resultados da viagem foram publicados (MILSTEAD, 1956) e os exemplares colhidos estão indicados neste artigo. Publicou ainda um artigo sobre Teídeos do Rio Grande do Sul (MILSTEAD, 1951).

As determinações de Milstead foram respeitadas, ressalvando as modificações taxonômicas advindas posteriormente. A maior dificuldade está no estabelecimento das subespécies por uma série de razões mas, principalmente, por encontrar-se no Rio Grande do Sul uma região central de transições faunísticas. Milstead não atacava subespécies, o que justificava, com razão, que a maioria das subespécies representantes neotropicais estava sem a devida fundamentação da análise populacional. A parte de Amphíbia foi examinada por nós mas, principalmente pelo autor júnior. Na parte de serpentes pôde-se anexar uma tabela (Tab. I) dos dados de grande parte dos exemplares, bem como anotações de captura porque $o$ autor sênior participou do colecionamento diretamente. Procuramos no Departamento mais dados, mas é bem possível que 
Tabela I. Dados dos exemplares de serpentes (parte) colhidas por W.W. Milstead (DMX=dentes maxilares; $\mathbf{D O}=$ filas de dorsais; $\mathbf{E X}=$ número do exemplar $(\mathrm{WWM}) ; \mathbf{F}=$ fêmea; $\mathbf{I L}=$ infralabiais; $\mathbf{M}=$ macho; $\mathbf{P R}=$ preocular; $\mathbf{P S}=$ postocular $\mathbf{S C}=$ subcaudais $; \mathbf{S E}=$ sexo $; \mathbf{S L}=$ supralabiais; $\mathbf{S V}+\mathbf{C C}=$ snout-vent [focinho-ânus] + comprimento caudal; $\mathbf{T T}=$ temporais; $\mathbf{V E}=$ ventrais $)$.

\begin{tabular}{|c|c|c|c|c|c|c|c|c|c|c|c|c|}
\hline ESPÉCIES & EX & $\mathrm{SV}+\mathrm{CC}$ & SE & DMX & VE & SC & DO & SL & IL & PR & PS & TT \\
\hline A. reticulatus ssp. & 506 & $293+48$ & $M$ & 8 & 140 & 31 & $15-15-15$ & 7 & 7 & 1 & 2 & $1+2$ \\
\hline C. bicarinatus & 1342 & $801+493$ & M & 27 & 158 & 150 & $12-12-10$ & $8 / 9$ & 10 & 1 & 2 & $1+1$ \\
\hline \multirow[t]{2}{*}{ C. rustica } & 490 & $1200+180$ & $M$ & $12+2$ & - & 56 & $? ?-19-17$ & 8 & 10 & 1 & 2 & $1+2$ \\
\hline & 960 & $1006+240$ & M & $11+2$ & 217 & 71 & $19-19-17$ & 7 & $9 / 8$ & 1 & 2 & $2+2$ \\
\hline E. (P.) I. trilineatus & 452 & $174+21$ & $\mathrm{~F}$ & $4+2$ & 187 & 34 & $15-15-15$ & 6 & 7 & 1 & 2 & $1+1$ \\
\hline \multirow[t]{5}{*}{ H. infrateniatus } & 153 & $345+109$ & $\mathrm{~F}$ & $15+2$ & 229 & 62 & $19+19+17$ & $7 / 8$ & 10 & 1 & 2 & $1+2$ \\
\hline & 188 & $156+51$ & $M$ & $15+2$ & 127 & 63 & $19-19-17$ & 8 & 10 & 1 & 2 & $1+2$ \\
\hline & 312 & $305+139$ & $M$ & $17+2$ & 122 & 74 & $19 \cdot 19 \cdot 17$ & $7 ?$ & $10 ?$ & 1 & 2 & $2+2$ \\
\hline & 479 & $598+130$ & M & $16+2$ & 137 & $53+$ & $19-19-17$ & 8 & 10 & 1 & 2 & $1+1$ \\
\hline & 1346 & $398+192$ & M & $17+2$ & 132 & 85 & $19-19-17$ & 7 & 10 & 1 & 2 & $1+2$ \\
\hline \multirow[t]{4}{*}{ L. almadensis } & 481 & $275+81$ & $M$ & $16+2$ & 153 & 64 & $19-19-17$ & 8 & 10 & 1 & 2 & $1+2$ \\
\hline & 701 & $348+90$ & $M$ & $16+2$ & 157 & 62 & $19 \cdot 19 \cdot 17$ & 8 & 10 & 1 & 2 & $1+2$ \\
\hline & 1094 & $380+97$ & $\mathrm{~F}$ & $15+2$ & 159 & 59 & $19 \cdot 19 \cdot 17$ & 8 & 10 & 1 & 2 & $1+2 / 1+1+2$ \\
\hline & 1122 & $273+72$ & $\mathrm{~F}$ & $17+2$ & 149 & 58 & $19-19-17$ & 8 & 10 & 1 & 2 & $1+2$ \\
\hline \multirow[t]{9}{*}{ L. anomalus } & 192 & $347+98$ & $\mathrm{~F}$ & $10+2$ & 157 & 60 & $19-19-15$ & 8 & 10 & 1 & 2 & $1+2$ \\
\hline & 193 & $289+76$ & $\mathrm{~F}$ & $11 ?+2$ & 143 & 58 & $19-19-15$ & $9 / 8$ & $8 / 10$ & 1 & 2 & $1+2$ \\
\hline & 263 & $451+100$ & $\mathrm{~F}$ & $13 ?+2$ & 150 & 51 & $19-19-15$ & 8 & 10 & 1 & 2 & $1+2$ \\
\hline & 264 & $298+87$ & $M$ & $12+2$ & 147 & 60 & $19-19-15$ & $8 / 9$ & 10 & 1 & 2 & $1+2$ \\
\hline & 265 & $369+160$ & $M$ & $12+2$ & 146 & $45+$ & $19-19-15$ & 8 & 10 & 1 & 2 & $1+2$ \\
\hline & 266 & $347+94$ & $\mathrm{~F}$ & $14+2$ & 147 & 55 & $19-19-15$ & 8 & 10 & 1 & 2 & $1+2$ \\
\hline & 306 & $277+93$ & $M$ & $12+2$ & 160 & 75 & $19-19-15$ & 8 & 10 & 1 & 2 & $1+2$ \\
\hline & 582 & $413+127$ & $\mathrm{~F}$ & $13+2$ & 149 & 70 & $19-19-15$ & 7 & 9 & 1 & 2 & $1+2$ \\
\hline & 1267 & $274+89$ & $\mathrm{~F}$ & $11+2$ & 148 & 68 & $19-19-17$ & 8 & $10 / 11$ & 1 & 2 & $1+2$ \\
\hline L. flavifrenatus & 961 & $515+185$ & $\mathrm{~F}$ & $20+2$ & 164 & 81 & $? ?-17-13$ & 8 & 10 & 1 & 2 & $2+2$ \\
\hline \multirow[t]{2}{*}{ L. j. jaegeri } & 149 & $309+93$ & $\mathrm{M}$ & $22+2$ & 150 & 68 & $17-17-17$ & 8 & 9 & 1 & 2 & $1+2$ \\
\hline & 585 & $236+74$ & $\mathrm{M}$ & $20+2$ & 154 & 62 & $17-17-17$ & 8 & 10 & 1 & 2 & $1+2$ \\
\hline \multirow[t]{3}{*}{ L. miliaris ssp. } & 190 & $462+95$ & $\mathrm{~F}$ & $15+2$ & 178 & 56 & $17 \cdot 17 \cdot 15$ & 8 & $10 / 9$ & 1 & 2 & $1+2$ \\
\hline & 897 & $617+138$ & $\mathrm{~F}$ & $13+2$ & 165 & 54 & $17-17-15$ & 8 & 10 & 1 & 2 & $1+2$ \\
\hline & 984 & $205+51$ & $\mathrm{M}$ & $15+2$ & 161 & 60 & $17-17-15$ & 8 & 10 & 1 & 2 & $1+2$ \\
\hline L. p. platensis & 300 & $310+63$ & $\mathrm{M}$ & $15+2$ & 148 & 44 & $19-19-15$ & 8 & 10 & 1 & 2 & $1+2$ \\
\hline L. $\underline{\text { p. subfasciatus }}$ & 308 & $468+91$ & $\mathrm{~F}$ & $15+2$ & 157 & 50 & $19 \cdot 19 \cdot 15$ & 8 & 10 & 1 & 2 & $1+2$ \\
\hline \multirow[t]{12}{*}{ L. poecilogyrus ssp. } & 18 & $309+72$ & $\mathrm{~F}$ & $14+2$ & 142 & 51 & $19-19-15$ & $8 / 9$ & 10 & 1 & 2 & $1+2$ \\
\hline & 19 & $335+77$ & $\mathrm{~F}$ & $14+2$ & 142 & 53 & $19-19-15$ & 8 & 10 & 1 & 2 & $1+2$ \\
\hline & 20 & $297+74$ & $\mathrm{M}$ & $15+2$ & 141 & 50 & $19-19-15$ & 8 & 10 & 1 & 2 & $1+2$ \\
\hline & 33 & $280+70$ & $\mathrm{M}$ & $15+2$ & 134 & 40 & $19-19-15$ & 8 & 10 & 1 & 2 & $1+2$ \\
\hline & 186 & $341+73$ & $\mathrm{~F}$ & $16+2$ & 141 & 44 & $19-19-15$ & 8 & 10 & 1 & 2 & $1+2$ \\
\hline & 371 & $244+54$ & F & $14+2$ & 146 & 49 & $19-19-15$ & 8 & 10 & 1 & 2 & $1+2$ \\
\hline & 498 & $464+91$ & $M$ & $14+2$ & 143 & 48 & $19 \cdot 19 \cdot 15$ & 8 & 10 & 1 & 2 & $1+2$ \\
\hline & 579 & $275+64$ & M & $16+2$ & 143 & 49 & $19-19-15$ & 8 & 10 & 1 & 2 & $1+2$ \\
\hline & 702 & $528+108$ & F & $15+2$ & 152 & $45+$ & $19-19-15$ & 8 & 10 & 1 & 2 & $1+2$ \\
\hline & 957 & $401+117$ & M & $16+2$ & 148 & 51 & $19-19-15$ & 8 & 10 & 1 & 2 & $1+2 / 1+1+2$ \\
\hline & 1227 & $369+107$ & $\mathrm{~F}$ & $18+2$ & 152 & 66 & $19 \cdot 19 \cdot 17$ & 8 & 10 & 1 & 2 & $1+2$ \\
\hline & 1264 & $278+61$ & $M$ & $16+2$ & 146 & 47 & $? ?-19-15$ & 8 & 10 & 1 & 2 & $1+2$ \\
\hline \multirow[t]{2}{*}{ L. dorbignyi ssp. } & 185 & $371+60$ & F & $6+1$ & 139 & 38 & $21-21-17$ & 7 & 10 & 1 & 2 & $1+2$ \\
\hline & 304 & $429+53$ & $M$ & $5+1$ & 134 & 27 & $21 \cdot 19 \cdot 17$ & 7 & 10 & 1 & 2 & $1+2$ \\
\hline
\end{tabular}

Cont. 
Tab. I (cont.)

\begin{tabular}{|c|c|c|c|c|c|c|c|c|c|c|c|c|}
\hline ESPÉCIES & $\mathrm{EX}$ & $\mathrm{SV}+\mathrm{CC}$ & $\mathrm{SE}$ & DMX & VE & $\mathrm{SC}$ & DO & SL & IL & PR & PS & TT \\
\hline \multirow[t]{5}{*}{ L. dorbignyi ssp. } & 307 & $393+72$ & $\mathrm{~F}$ & $4+1$ & 134 & 31 & $21-21-17$ & 7 & $9 / 10$ & 1 & 2 & $1+2$ \\
\hline & 480 & $374+69$ & M & $6+1$ & 136 & 40 & $21-21-17$ & 7 & 9 & 1 & 2 & $1+2$ \\
\hline & 581 & $445+62$ & $\mathrm{~F}$ & $6+1$ & 134 & 33 & $21 \cdot 21 \cdot 17$ & 7 & 9 & 2 & $3 / 2$ & $1+2$ \\
\hline & 700 & $333+61$ & M & $5+1$ & 139 & 40 & $21 \cdot 21 \cdot 17$ & 7 & 9 & 2 & 2 & $1+2$ \\
\hline & 1265 & $326+47$ & $\mathrm{~F}$ & $4+2$ & 129 & 32 & $21-21-17$ & 7 & 9 & 1 & 2 & $1+2$ \\
\hline M. bifossatus & 1270 & $930+331$ & M & 27 & 173 & 89 & $15 \cdot 15 \cdot 15$ & 8 & 10 & 1 & 2 & $2+2$ \\
\hline \multirow[t]{2}{*}{ M. frontalis ssp. } & 489 & $271+16$ & $\mathrm{~F}$ & - & 217 & 18 & $15-15-15$ & 7 & 7 & 1 & 2 & $1+1$ \\
\hline & 959 & $510+42$ & M & - & 201 & 23 & $15-15-15$ & 7 & 7 & 0 & 2 & $1+1$ \\
\hline O. rhombifer ssp. & 482 & $433+121$ & M & $10+2$ & 183 & 75 & $19-19-17$ & 8 & 10 & 1 & 2 & $2+2$ \\
\hline \multirow[t]{2}{*}{$\underline{\mathrm{P}}$ a. subcarinatus } & 32 & $808+253$ & $\mathrm{~F}$ & $13+2$ & 197 & 93 & $21 \cdot 21 \cdot 17$ & $9 / 8$ & $10 / 11$ & 1 & 2 & $1+2$ \\
\hline & 503 & $853+344$ & $\mathrm{~F}$ & $13+2$ & 196 & 126 & $21-21-17$ & 8 & 11 & 1 & 2 & $1+2$ \\
\hline \multirow[t]{4}{*}{ P. o. olfersii } & 42 & $413+150$ & $\mathrm{~F}$ & $10+2$ & 208 & 125 & $19 \cdot 19 \cdot 15$ & 8 & $11 / 10$ & 1 & 2 & $1+2$ \\
\hline & 111 & $403+163$ & $\mathrm{~F}$ & $11+2$ & 203 & 126 & $19-19-15$ & 8 & 9 & 1 & 2 & $1+2$ \\
\hline & 587 & $780+340$ & M & $10+2$ & 187 & 116 & $21-19-15$ & $9 / 8$ & 11 & 1 & 2 & $1+2$ \\
\hline & 962 & $664+256$ & $\mathrm{~F}$ & $10+2$ & 198 & 117 & $19-19-15$ & 8 & 11 & 1 & 2 & $2+3 / 1+2$ \\
\hline \multirow[t]{13}{*}{ P. patagoniensis } & 203 & $641+248$ & M & $9+2$ & 170 & 95 & $19-19-15$ & 7 & 9 & 1 & 2 & $3+2$ \\
\hline & 580 & $759+244$ & $\mathrm{~F}$ & $9+2$ & 184 & 94 & $19 \cdot 19 \cdot 15$ & 7 & 9 & 1 & 2 & $1+2$ \\
\hline & 583 & $698+390$ & M & $10+2$ & 183 & 91 & $19 \cdot 19 \cdot 15$ & 7 & 9 & 1 & 2 & $2+3$ \\
\hline & 584 & $659+262$ & M & $9+2$ & 164 & 90 & $19-19-15$ & 7 & 9 & 1 & 2 & $1+2$ \\
\hline & 586 & $609+261$ & M & $9+2$ & 175 & 108 & $19-19-15$ & 7 & 9 & 1 & 2 & $1+2$ \\
\hline & 898 & $756+225$ & $\mathrm{~F}$ & $10+2$ & 185 & 84 & $19 \cdot 19 \cdot 15$ & 7 & 9 & 1 & 2 & $1+2$ \\
\hline & 899 & $968+286$ & $\mathrm{~F}$ & $9+2$ & 183 & $84+$ & $19 \cdot 19-15$ & 7 & 9 & 1 & $3 / 2$ & $1+2$ \\
\hline & 902 & $667+280$ & M & $10+2$ & 169 & 103 & $19-19-15$ & 7 & $9 / 10$ & 1 & 2 & $1+2$ \\
\hline & 958 & $595+247$ & M & $10+2$ & 171 & 108 & $19-19-15$ & 7 & 9 & 1 & 2 & $1+2$ \\
\hline & 985 & $815+276$ & $\mathrm{~F}$ & $9+2$ & 182 & 91 & $18-18-15$ & 7 & 9 & 1 & 2 & $1+2$ \\
\hline & 988 & $690+229$ & $\mathrm{~F}$ & $10+2$ & 183 & 95 & $19-19-15$ & 7 & 9 & 1 & 2 & $1+2$ \\
\hline & 1263 & $723+250$ & $\mathrm{~F}$ & $10+2$ & 188 & 86 & $19 \cdot 19 \cdot 15$ & 7 & 9 & 1 & 2 & $1+2$ \\
\hline & 1269 & $274+84$ & $\mathrm{~F}$ & $10+2$ & 185 & 88 & $? ?-19-15$ & 7 & 9 & 1 & 2 & $1+2$ \\
\hline R. occipitalis & 1268 & $328+101$ & $\mathrm{~F}$ & $14+2$ & 183 & 70 & $15-15-15$ & 8 & 9 & 1 & 2 & $2+2$ \\
\hline R. poecilopogon & 1487 & $128+47$ & $\mathrm{~F}$ & $14+2$ & 151 & 71 & $17 \cdot 17 \cdot 17$ & 7 & 8 & 1 & 2 & $1+2$ \\
\hline \multirow[t]{5}{*}{ S. ventrimaculatus } & 372 & $507+93$ & $\mathrm{~F}$ & 10 & 160 & 50 & $15 \cdot 15 \cdot 15$ & 6 & 8 & 0 & 2 & $1+1$ ou $2+0$ \\
\hline & 483 & $359+95$ & M & 12 & 157 & 49 & $15-15-13$ & 8 & 10 & 0 & 2 & $1+2$ \\
\hline & 954 & $381+93$ & $\mathrm{M}$ ? & 14 & 165 & 59 & $15 \cdot 15 \cdot 15$ & 7 & 8 & 1 & 2 & $1+2$ \\
\hline & 955 & $446+103$ & $\mathrm{~F} ?$ & 10 & 161 & 45 & $15 \cdot 15 \cdot 15$ & $7 / 6$ & 8 & 1 & 2 & $1+2 / 2+1$ \\
\hline & 956 & $372+98$ & $\mathrm{M}$ ? & 12 & 160 & 55 & $? ?-15-15$ & 7 & - & 1 & 2 & $1+2 / 1+1$ \\
\hline T. dorsatus & 112 & $516+153$ & M & $5+2$ & 138 & 59 & $17-17-15$ & 7 & 9 & 1 & 2 & $1+2$ \\
\hline \multirow[t]{5}{*}{ T. $\overline{\text { ocelatus }}$} & 191 & $325+49$ & $\mathrm{~F}$ & $5+2$ & 133 & 28 & $19-19-17$ & 7 & $9 / 10$ & 1 & 1 & $1+2$ \\
\hline & 301 & $278+48$ & $\mathrm{M}$ & $7+2$ & 139 & 38 & $19-19-15$ & 7 & $9 / 10$ & 1 & 2 & $2+2$ \\
\hline & 302 & $437+46$ & $\mathrm{~F}$ & $5+2$ & 144 & 33 & $19 \cdot 19 \cdot 17$ & 7 & 9 & 1 & 1 & $1+2$ \\
\hline & 303 & $309+57$ & $\mathrm{M}$ & $6+2$ & 142 & 37 & $19-19-17$ & 7 & 9 & 1 & 2 & $2+2 / 1+2$ \\
\hline & 305 & $287+44$ & $\mathrm{~F}$ & $5+2$ & 139 & 37 & $18 \cdot 18 \cdot 15$ & 7 & 9 & 1 & 2 & $1+2$ \\
\hline \multirow[t]{2}{*}{ M. merremi } & 56 & $514+107$ & $\mathrm{M}$ & $5+1$ & 132 & 44 & $19-19-17$ & 7 & 11 & 2 & 3 & $1+2$ \\
\hline & 1266 & $725+84$ & $\mathrm{~F}$ ? & $7+1$ & 139 & 28 & $? ?-? ?-? ?$ & 7 & - & 1 & 3 & $1+3 / 1+2$ \\
\hline
\end{tabular}

Milstead tenha levado consigo.

Chamamos a atenção dos curadores das coleções de Instituições que receberam exemplares, para as possíveis alterações aqui introduzidas, tanto na identificação como nos dados de coleta, tendo em vista os apontamentos do autor sênior. 


\section{MATERIAL E MÉTODOS}

\section{Coleta}

Milstead colecionou quase todos os espécimens encontrados ou doados, mesmo os exemplares muito amassados pelo tráfego nas estradas, tendo anotado as ocorrências daqueles que foram desprezados. Esses apontamentos não encontramos. Ele costumava sair a rodar em marcha lenta $(40-50 \mathrm{Km} / \mathrm{h})$ e ia anotando, ora "D.O.R." (Dead On Road) ora "A.O.R." (Active On Road), mais para serpentes, que são os répteis mais encontrados em rodovias. Costumava sair quando o tempo estava para chover, de dia ou de noite, ou após fortes chuvas de verão quando saía sol. Nos acampamentos usava uma rede-barraca do exército dos E.U.A., agilizando a montagem e desmontagem. Cada espécimen obtido era lavado, aparados os órgãos exteriorizados, injetando formalina a $10 \%$, etiquetado e posto a fixar, passando os apontamentos para o caderno de campo que, no laboratório, eram transportados para fichas específicas; a etiqueta de campo era cadarço impresso com carimbo numerador e tinta a óleo, fixa; o da coleção era uma chapinha de metal (liga com alumínio) e impressa com tipos. Os exemplares vivos eram mortos na hora, para reter provável alimento no estômago. As etiquetas metálicas de Milstead vieram a oxidar posteriormente, demonstrando não ter sido uma boa liga. Nós substituimos essas etiquetas por fita plástica gravada com rotulador.

\section{Deposição}

A coleção foi tombada com a sigla "WWM", iniciais do colecionador, sendo os exemplares enfrascados conforme a espécie. Antes de partir Milstead selecionou exemplares de todas as espécies colhidas para diferentes instituições, a seguir relacionadas juntamente com o nome dos respectivos curadores naquela ocasião.

FMNH, Field Museum of Natural History, Chicago, Illinois, U.S.A. (Dr. Karl Patterson Schimdt, falecido).

MNRJ, Museu Nacional da Universidade Federal do Rio de Janeiro, Rio de Janeiro, Brasil (Prof. Antenor Leitão de Carvalho, falecido).

MZUSP, Museu de Zoologia de Universidade de São Paulo, São Paulo, Brasil (Dr. Paulo Emílio Vanzolini).

UFRGS, Museu do Departamento de Zoologia do. Instituto de Biociências da Universidade Federal do Rio Grande do Sul, Porto Alegre, Rio Grande do Sul, Brasil (Dr. Celso Paulo Jaeger).

\section{Auxiliares}

Os principais colaboradores no colecionamento são citados por siglas, tendo em vista evitar a repetição cansativa e o alongamento do texto:

$\mathbf{A c}=$ Acadêmico(s) do ex-curso de História Natural da UFRGS.

ARC $=$ Dr. Antonio Rodrigues Cordeiro, UFRGS (atualmente na Universidade Federal do Rio de Janeiro). 
$\mathbf{B Z L}=$ Dr. Breno Zanella de Lema (irmão do autor sênior).

CPJ $=$ Dr. Celso Paulo Jaeger, UFRGS.

EWG $=$ Prof. Eugênio Wedelstaedt Gruman, UFRGS.

FT $=$ Dra. Flávia Tórgo (UFRGS).

$\mathbf{J A P}=$ Dr. Jorge Alberto Petersen (UFRGS, falecido).

$\mathbf{P F}=$ Dr. Paulo Friedmann, UFRGS (atualmente na Universidade Federal de Rio Grande, RS).

TDL $=$ Thales de Lema, UFRGS (atualmente na Pontíficia Universidade Católica do Rio Grande do Sul, RS).

$\mathbf{W W M}=$ Dr. William Wright Milstead.

\section{Localidades}

As localidades mais citadas são indicadas por siglas, de preferência aquelas de uso rodoviário: AR, Argentina; BR, Brasil; PR, Estado Paraná, BR; RS, Estado Rio Grande do Sul, BR; SC, Estado Santa Catarina, BR; SP, Estado São Paulo, BR.

\section{Medidas}

Apresentadas em milímetro e somente as de comprimento, sendo: SV ("snout-vent"), do focinho à cloaca; $\mathrm{CC}$, cauda.

\section{INDICAÇÕES GEOGRÁFICAS}

Todas as citações geográficas políticas são aqui indicadas em ordem alfabética e dados de sua localização, conforme a figura 1, dispensando a exaustiva citação das coordenadas.

\section{ARGENTINA}

Buenos Aires: Província cuja capital situa-se às margens do Rio de La Plata:

- Azul: coletas a $135 \mathrm{Km}$ a NE e $92 \mathrm{Km}$ a SW.

- Bahía Blanca: a 17, 19 e 20Km a W.

- Dolores: região do Canal I, 1 a 3, 4 e 25Km.

- Magdalena: a 20Km S.

- Pergamino: a $121 \mathrm{Km}$ SE.

- Villa General Conesa: em fazenda.

Entre Rios: Província adjacente ao RS:

- Paraná: a 17, 20, 58, 62 e 90Km a NE.

Santa Fe: Província junto ao Rio Paraná:

- Maciel: a $10 \mathrm{Km} \mathrm{S.}$ 


\section{BRASIL}

Excetuando São Paulo, do qual há raros espécimens, os demais procedem dos três estados do sul, principalmente do Rio Grande do Sul.

\section{Paraná:}

- Ribeirão Claro: município a NE, onde o autor sênior residiu e colecionou material herpetológico.

\section{Santa Catarina:}

- Florianópolis: na Ilha de Santa Catarina.

\section{Rio Grande do Sul:}

- Bento Gonçalves: planalto oriental, junto ao Rio das Antas.

- Canoas: Niterói: junto a Grande Porto Alegre, ao N.

- Capão da Canoa: litoral Atlântico norte:

- Atlândida: balneário ao sul.

- Lagoa dos Quadros: lagoa costeira entre o litoral e BR 101, junto à Capão da Canoa.

- Carlos Barbosa: no planalto oriental, ao sul de B. Gonçalves.

- Caxias do Sul: no planalto oriental:

- Ana Rech: ao norte da cidade de Caxias.

- Dom Pedrito: na serra de sudeste.

- Farroupilha: planalto oriental, próximo à B. Gonçalves, coletas a 18, 32 e $41 \mathrm{Km} \mathrm{S}$.

- Garibaldi: planalto oriental, próximo à B. Gonçalves.

- Gravataí: na planície costeira, sendo montanhosa ao n; coletas a 4 e $49 \mathrm{Km} \mathrm{NE}$; a 12,13 e $16 \mathrm{Km}$ E.

- Guaíba: junho ao delta do rio Jacuí, para W; coletas a 14, 19, 44, 63Km W; saindo de Porto Alegre.

- Montenegro: na encosta inferior do planalto oriental:

- Barão: ao sul da sede municipal, cerca de $400 \mathrm{~m}$ de altitude.

- Nova Petrópolis: planalto oriental, próxima à Canela.

- Osório: local de maiores coletas foi a $10 \mathrm{Km} \mathrm{S}$, chamado Emboabas, na planície costeira; outros: 10 e 19Km NE; 2, 4, 8 e 16Km a SE. Essa área é rica de lagoas, destacando-se a Lagoa dos Barros e do Lessa.

- Porto Alegre: com a capital estadual, entre a Depressão Central e a encosta inferior do Planalto a NE:

- Bela Vista: bairro da capital.

- Bom Fim: ídem.

- Floresta: ídem.

- Menino Deus: ídem.

- Petrópolis: ídem, próximo à Bela Vista.

- Bairros afastados nas elevações graníticas e areníticas:

- Agronomia: contígua à Viamão.

- Glória: junto ao Morro da Polícia. 
- Teresópolis: junto ao Morro da Cruz e Vila Nova.

- Bairros juntos ao sistema flúvio-lacustre do Rio Guaíba e Laguna dos Patos:

- Belém Novo.

- Ipanema: Morro do Osso.

- Itapuã.

- Ponta Grossa: Morro da Ponta Grossa.

- São Pedro: Morro de São Pedro.

- Serraria: junto à Ponta Grossa.

- Tristeza: região mais povoada.

- Rio Pardo: orla sul do Planalto Central.

Bexiga: a $39 \mathrm{Km} \mathrm{W}$.

- Outros pontos no município: $21,26,34,44,50,52,54,79$ e $128 \mathrm{Km}$ E, e $30 \mathrm{Km} \mathrm{W}$.

- Santa Maria: parte na borda sul do planalto e parte na depressão; coletas na região da sede e à $25 \mathrm{Km} \mathrm{SE}$.

- Santo Antônio da Patrulha: entre a planície costeira e o planalto; pontos a $19 \mathrm{Km}$ E e $30 \mathrm{Km}$ SW.

- São Francisco de Paula: no planalto oriental a NE; pontos a 2, 11 e $30 \mathrm{Km} \mathrm{NE}$, e a $21 \mathrm{Km} \mathrm{SW}$.

- São Leopoldo: ao N de P. Alegre, coleta a $5 \mathrm{Km} \mathrm{N}$.

- São Vicente do Sul: a W do Estado

- General Vargas: a 36Km E.

- Torres: litoral norte, parte costeira e parte na escarpa do planalto:

- Lagoa Itapeva: a NW.

- Tramandaí: litoral centro-N:

- Cidreira: balneáreo ao sul de Tramandaí.

- Imbé: balneáreo junto à sede.

- Lagoa do Lessa: no interior, junto à Osório.

- Uruguaiana: fronteira com Argentina; local a $20 \mathrm{Km}$ da cidade.

- Viamão: a E de P. Alegre; para o S, junto à Laguna dos Patos:

- Lomba do Sabão: próximo a P. Alegre.

- Morro do Côco: a 50Km SE, junto ao rio; densamente florestada.

- Tarumã: campos ondulados.

\section{LISTA SISTEMÁTICA}

Apenas os táxons de categoria superior seguem a ordem sistemática tradicional. Os gêneros e espécies estão em ordem alfabética face ao estágio de conhecimento em que se encontra a filogenia dos táxons e as modificações constantes que vêm sofrendo em resultado de recentes estudos.

Os gêneros estão numerados em algarismos romanos, e as espécies em arábicos, ambos em sequência corrida dentro de cada classe. 
Os dados apresentados seguem a ordem: procedência - número do exemplar - sigla da Instituição a que foi doado - data de entrada ou coleta nome (sigla) coletor(es). A numeração é de W.W. Milstead. A sistemática de Anura segue LAURENT (1967) e a de Gymnophiona, de TAYLOR (1968). A de répteis segue a de DOWLING \& DUELLMAN (1978). A de Sauria está atualizada por ESTES \& PREGILL (1988) e FROST \& ETHERIDGE (1989). $\mathrm{O}$ autor Braun não aceita o gênero Ololygon.

\section{AMPHIBIA}

GYMNOPHIONA

TYPHLONECTIDAE

\section{Chthonerpeton Peters, 1879}

1. Chthonerpeton indistinctum (Reinhardt \& Lütken, 1862)

RS - Porto Alegre (sede): 574 (UFRGS) - 10.1941, TDL.

Observação. Encontrada à noite, na rua João Alfredo, durante a enchente do Rio Guaíba.

\section{ANURA \\ MICROHYLOIDAE MICROHYLIDAE \\ II. Elachistocleis Parker, 1927}

2. Elachistocleis bicolor (Valenciennes, 1838)

RS - Carlos Barbosa: 527/9 (MZUSP), 530/2 (FMNH), 533/5 (MNRJ) I-1947, TDL. Farroupilha (18Km S): 431 (FMNH), 500/1 (UFRGS) - 30-III-54 WWM. Gravataí (49Km NE): 1061 (MNRJ), 1062 (FMNH) - 26-XI-55, WWM \& JAP. Osório (Emboabas): 896 (MZUSP) - 21-II-54, WWM \& JAP. Porto Alegre (Teresópolis): 953 (FMNH) - 29-XII-54, WWM \& JAP; 1163 (UFRGS) - 9-I-55, TDL \& EWG. Rio Pardo (37Km W): 327 (FMNH) - 25-II-54, WWM.

\section{BUFONOIDEA \\ LEPTODACTYLIDAE}

\section{Hylodes Fitzinger, 1826}

\section{Hylodes meridionalis (Mertens, 1927)}

RS - São Francisco de Paula 21 (Km SW): 900 (UFRGS), 901 (FMNH) - 14-XI-54, WWM. 


\section{Leptodactylus Fitzinger, 1826}

4. Leptodactylus fuscus (Schneider, 1799)

RS - Rio Pardo (Bexiga): 598, 979/80 (MNRJ), 599, 978 (FMNH), 672/3 (UFRGS), 674/5 (MZUSP) - 5-II-54, WWM \& JAP.

5. Leptodactylus gracilis Duméril \& Bibron, 1841

RS - Carlos Barbosa: 538/9 (MNRJ), 540/1 (FMNH) - 1-1947, TDL \& BZL. Osório (Emboadas): 162, 174 (MZUSP) - 8-I-54, WWM; 706 (UFRGS), 811/9 (MNRJ), 820/7, 856 (FMNH) - 21-XI-54, WWM \& JAP. Porto Alegre (Teresópolis): 1187 (MNRJ) - 9-I-55, TDL \& EWG. Rio Pardo (Bexiga: 600 (UFRGS) - 5-XI-54, WWM \& TDL. Torres: 30 (MNRJ) - 6-XI-53, CPJ. Tramandaí (Imbé): 86 (FMNH) - 20-XII-53, WWM.

\section{Leptodactylus latinasus latinasus Espada, 1875}

RS - Porto Alegre (Bela Vista): 707/10 (FMNH) - 9-XI-54, JAP; (Morro da Polícia): 2 (UFRGS) - 21-X-53, WWM, 578 (MNRJ) - 5-II-54, Ac; (Teresópolis): 1188/93 (FMNH), 1194/9 (MNRJ) - 9-I-55, TDL \& EWG. Rio Pardo: 341 (MNRJ), 369/70 (MZUSP) - 25-II-54, WWM; 671 (MZUSP) - 6-XI-54, JAP. Rio Pardo (Bexiga): 971/7 (MNRJ) - 14-I-55, WWM. Uruguaiana (20Km E): 189 (FMNH) - 23-I-53, WWM. Viamão (Morro do Côco): 125 (UFRGS) 26-XII-53, TDL, PF \& EWG.

\section{Leptodactylus mystacinus (Burmeister, 1861)}

RS - Carlos Barbosa: 537 (MNRJ) - I-1947, TDL. Farroupilha: 432 (MNRJ), 433 (FMNH) - 30-III-54, WWM. Rio Pardo (Bexiga): 699 (UFRGS), 1128, 1214/25 (MNRJ), 1153/4 (FMNH), 1200/1 (MZUSP) - 7-XI-55, WWM \& TDL.

\section{Leptodactylus ocellatus (Linnaeus, 1758)}

RS - Capão da Canoa: 1250/3 (FMNH) - 20.11.54, WWM. Carlos Barbosa: 536 (MNRJ) - I-1947, TDL. Farroupilha (18Km S): 997 (FMNH) - 7-II-55, ARC. Osório (Emboadas): 16 (FMNH) - 24-X-53, WWM; 175 (FMNH) 8-I-54, WWM; 436 (FMNH) - 5-IV-54, WWM; 782, 841/7 (MNRJ), 809/10, 848/55 (FMNH) - 20-XI-54, WWM; 998/1005 (MNRJ), 1000/13 (FMNH) 25-XI-55, WWM \& JAP; 1105/7, 1113/4 (FMNH), 1108/9, 1115/7, 1246/9 (MNRJ) - 5-III-55, JAP. Porto Alegre (Bela Vista): 704/5 (FMNH) - 9-XI-54, WWM \& JAP; (Belém Novo): 1369 (FMNH) - XII-1941, TDL \& W.A. Schimth; (Serraria): 1081 (MNRJ) - 28-III-55, WWM; 1271/6 (MNRJ) - 28-II-55, EWG; (Teresópolis): 34 (MNRJ) - 12-XI-53, WWM; 309 (MNRJ) - 22-II-54, TDL \& EWG; 913/7 (UFRGS), 981 (FMNH) - 29-XII-54, WWM \& TDL. Rio Pardo (Bexiga): 54 (FMNH) - 28-XI-53, WWM; 342 (FMNH), 367/8 (MNRJ) 25-II-54, WWM; 590/3 (FMNH), 594/7 (MNRJ), 676/7 (MZUSP) - 5-II-54, JAP \& ARC. Tramandaí (Imbé): 88/91 (FMNH) - 20-XII-53, Ac. Viamão (Morro do Côco): 122, 1377 (MNRJ) - 26-XII-53, WWM \& TDL.

AR - Buenos Aires: Bahía Blanca (17Km W): 249/50 (MNRJ), 251 
(FMNH) - 3-II-54, WWM \& ARC; Dolores: 272/9 (MNRJ) - 6-II-54, WWM \& ARC. Villa General Conesa: 265 (MNRJ) - 6-II-54, WWM \& ARC.

\section{Limnomedusa Cope, 1866}

9. Limnomedusa macroglossa (Duéril \& Bibron, 1841)

RS - Carlos Barbosa: 544 (FMNH), 545 (UFRGS) - I-1947, TDL.

\section{Odontophrynus Reinhardt \& Lütken, 1862}

10. Odontophrynus americanus (Duméril \& Bibron, 1841)

RS - Capão da Canoa: 1233, 1238 (MZUSP) - 12-III-55, JAP. Carlos Barbosa: 542 (MNRJ) - I-1947, TDL; 575 (MNRJ) - 11-I-46, TDL. Osório (Emboadas): 8/9 (MNRJ), 21/2 (FMNH) - 24-X-53, WWM \& CPJ; 439/40 (MNRJ), 453 (FMNH) - 5-IV-54, WWM 778 (FMNH) - 20-XI-54, WWM; 803/4 (UFRGS), 805/6, 1028 (MNRJ), 807/8 (FMNH) - 21-XI-54, WWM \& JAP; 1095 (FMNH) - 5-II-55, WWM \& JAP. Porto Alegre (Serraria): 554 (FMNH) - 5-X-52, TDL; (Teresópolis): 557 (MNRJ) - 12-V-51, TDL; 558 (FMNH) - I-1943, TDL; 559 (MNRJ) - IV-1943, TDL; 571 (FMNH) - VI-1951, TDL. Rio Pardo (Bexiga): 47 (MNRJ), 55 (FMNH) - 28-XI-53, WWM. Tramandaí (Imbé): 85 (MNRJ) - 20-XII-53, WWM \& J. L. Milstead. Viamão (Morro do Côco): 124(FMNH) - 26-XI-53, TDL, PF \& EWG.

\section{Physalaemus Fitzinger, 1826}

\section{Physalaemus biligonigerus (Cope, 1860)}

RS - Capão da Canoa (Atlântida): 576 (MNRJ) - 28-XII-52, M. Z. de Lema \& TDL. Carlos Barbosa: 543 (FMNH) - I-1947, TDL. Gravataí: 1053/6, 1063/4 (FMNH), 1057/9, 1065/6 (MNRJ) - 26-II-55, WWM \& JAP. Osório (Emboadas): 7 (MNRJ) - 24-X-53, WWM; 438, 446/50 (FMNH), 441/5 (MNRJ) - 5.4.54, WWM; 455/63 (MNRJ), 464/61 (FMNH) - 6-IV-54, WWM; 757/9, 779 (FMNH), 760/2 (MNRJ) - 20-XI-54, WWM; 784, 796/800 (MNRJ), 790/5 (FMNH) - 21-XI-54, WWM \& JAP; 1039 (FMNH) - 25-II-55, WWM \& JAP; 1516 (UFRGS) - 24-V-55, WWM. Porto Alegre (Bela Vista): 716/25 (MNRJ), 726/34 (FMNH) - 9-XI-54, WWM \& JAP; 903 (UFRGS), 904/5 (MZUSP) 28-XII-54; (Teresópolis): 310/1 (FMNH) - 22-II-54, WWM \& TDL; 556 (FMNH) - 12-V-51, TDL; 918 (MNRJ), 920/1 (FMNH), 922 (UFRGS) 29-XII-54, WWM \& TDL; (Morro São Pedro): 1511 (UFRGS) - O. A. Pereira. Santa Maria: 963 (MNRJ) - 13-I-55, WWM. Tramandaí (Imbé): 84 (FMNH) 20-XII-53, WWM. Viamão (Morro do Côco): 123 (MNRJ - 26-XII-53, TDL, PF \& EWG.

12. Physalaemus cuvieri Fitzinger, 1826

RS - Caxias do Sul (Ana Rech): 1344 (FMNH) - 8-IV-55, FT. Osório (2Km SE): 1126 (MNRJ) - 6-III-55, WWM; (Emboabas): 54 (MNRJ) - 5-IV-54, WWM; 765, 801/2, 859/61 (FMNH), 772/3, 857/8 (MNRJ) - 20-XI-54, WWM \& 
JAP; 1029 (FMNH), 1034 (MNRJ) - 25-II-55, WWM \& JAP; Porto Alegre (Bela Vista): 713/4 (MZUSP) - 9-XI-54, WWM \& JAP; (Agronomia): 1384 (MNRJ) - 22-II-53, EWG. Rio Pardo (Bexiga): 41 (FMNH), 51 (MNRJ) 28-XI-53, WWM; 334 (FMNH) - 25-II-54, WWM; 353/4 (UFRGS) - 25-II-54, WWM; 607 (FMNH) - 5-XI-54, WWM, JAP \& ARC; 631/7, 698 (MNRJ), 638/44 (FMNH) - 6-XI-54, JAP; 967 (MNRJ) - 14-I-55, WWM; 1127 (MNRJ), 1156/7 (FMNH) - 14-IV-54, WWM. Torres (Itapeva): 1509 (UFRGS) 30-IX-54, O. A. Pereira.

\section{Physalaemus gracilis (Boulenger, 1883)}

RS - Canoas (Niterói): 547/9 (MNRJ), 550/1 (FMNH) - 29-VII-51, WWM. Carlos Barbosa: 523/4 (FMNH), 525/6 (MNRJ) - I-1947, TDL. Caxias do Sul (Ana Rech): 1343 (MNRJ) - 8-IV-55, EWG. Farroupilha: 58/9 (FMNH), 60 (MNRJ) - 12-XII-53, WWM; 425/6 (FMNH), 427/8, 434 (MNRJ) - 29-III-54, WWM \& F. M. Salzano; 491/3, 502 (MNRJ), 494/6 (FMNH) - 3-V-54, WWM; 1347 (FMNH) - 20-III-55, WWM. Osório (Emboabas): 5/6, 11 (MNRJ) 24-X-53, WWM; 176/7 (FMNH) - 9-I-54, WWM; 451 (FMNH) - 5-IV-54, WWM; 572 (MNRJ) - 17-VI-51, WWM; 766 (UFRGS) - 767/9 (FMNH), 779/1 (MNRJ) - 20-XI-54, WWM \& JAP. Rio Pardo (Bexiga): 35/7 (FMNH), 38/40, 52 (MNRJ) - 28-XI-53, WWM; 356/7 (MZUSP) - 26-XI-54, WWM. Viamão (Morro do Côco): 128/34, 136/7, 555 (FMNH), 138/40, 1379 (MNRJ) 26-XII-53, TDL, PF \& EWG.

14. Physalaemus henseli (Peters, 1870)

RS - Rio Pardo (Bexiga): 589 (FMNH), 630 (UFRGS) - 5-XI-54, WWM, JAP \& ARC; 966 (FMNH) - 14-I-55, WWM; 1130 (FMNH), 1131 (MZUSP), 1155 (UFRGS) - 16-IV-55, WWM. Viamão (Morro do Côco): 126 (FMNH), 127, 135, 1378 (MNRJ) - 26-XII-53, TDL, PF \& EWG.

15. Physalaemus riograndensis Milstead, 1960

RS - Osório (4Km SE): 1940/5 (FMNH), 1046/51 (MNRJ), 1052 (MZUSP) - 26-II-55, WWM \& JAP. Rio Pardo (Bexiga): 315 (MZUSP), 326, 340 (UFRGS), 363/4 (FMNH), 365 (MNRJ) - 25-II-54, WWM. Santa Maria (25 Km SE): 964 (FMNH), 965 (MNRJ) - 13-I-55, WWM.

\section{Pleurodema Tschudi, 1838}

16. Pleurodema bibronii Tschudi, 1838

RS - Farroupilha (18Km S): 499 (UFRGS) - 11-V-54, WWM.

\section{Pseudopaludicola Miranda-Ribeiro, 1926}

17. Pseudopaludicola falcipes (Hensel, 1867)

RS - Osório (Emboabas): 15 (FMNH) - 24-X-53, WWM; 437 (MNRJ) - 5-IV-54, WWM; 1031 (MNRJ) - 25-II-55, WWM \& JAP; (4Km SE): 1035, 1037/8 (FMNH), 1036 (MNRJ) - 26-II-55, WWM \& JAP. Porto Alegre (Bela 
Vista): 715 (FMNH), 828 (MNRJ) - 9-XI-54, WWM \& JAP; (Belém Novo): 1370/3 (MNRJ), 1374 (FMNH) - XI-1941, W. A. Schmith \& TDL; (Teresópolis): 1167/76 (MNRJ), 1177/86 (FMNH) - 9-I-55, EWG. Rio Pardo (Bexiga): 313/4, 323/5, 366 (FMNH), 317/22 (MNRJ) - 25-II-54, WWM; 601/6 (UFRGS) - 5-XI-54, WWM, JAP \& ARC; 645/57 (MNRJ), 658/70 (FMNH - 6-XI-54, WWM \& JAP; 968/70 (MZUSP) - 14-I-55, WWM; 1137/44 (FMNH), 1145/51 (MNRJ) - 16-IV-55, WWM \& JAP. Torres: 1510 (UFRGS) - 1-XI-54, O. A. Pereira. Viamão (Morro do Côco): 1381/2 (FMNH) - 26-XII-53, TDL.

\section{HYLIDAE}

\section{Hyla Laurenti, 1768}

18. Hyla altera Lutz, 1968

RS - Osório (Emboabas): 23/4 (MZUSP) - 27-XI-53, WWM; 154/60 (MNRJ) - 7-I-54, WWM; 167/8 (FMNH) - 8-I-54, WWM; 472/8 (FMNH) 6-IV-54, WWM \& DPJ; 577 (MNRJ) - 3-IV-53, L. Buckup; 763/4 (MNRJ), 775/7 (UFRGS), 830 (FMNH) - 20-XI-54, WWM \& JAP; 1030 (MNRJ) 25-II-55, WWM \& JAP. Torres (Itapeva): 31 (FMNH) - 5-XI-53, CPJ.

19. Hyla faber Wied, 1821

RS - Farroupilha (18Km S): 991/3 (MNRJ) - 7-II-55, ARC Porto Alegre (Serraria): 1077/80, 1326/33 (FMNH), 1334/41 (MNRJ) - 28-II-53, WWM, CPJ, EWG, FT \& O. A. Pereira; (Teresópolis): 949 (MNRJ), 950 (FMNH), 951 (UFRGS), 952 (MZUSP) - 29-XII-54, WWM.

20. Hyla fuscovaria (Lutz, 1925)

RS - Carlos Barbosa: 507/15 (MNRJ) - 11-I-46, TDL. Osório (Emboabas): 774 (FMNH), 871 (MNRJ) - 20-XI-53, WWM \& JAP. Porto Alegre (Agronomia): 1385/6 (FMNH) - 22-XI-53, EWG; (Bela Vista): 906/12 28-XII-54, WWM; (Morro da Polícia): 3 (MNRJ) - 21-XI-53, WWM; (Morro São Pedro): 1345 (MNRJ) - 17-IV-55, WWM; (Teresópolis): 570 (FMNH) 29-IV-51, TDL; 939/43 (FMNH), 944/8 (MNRJ) - 29-XII-54, WWM \& TDL. Rio Pardo (Bexiga): 329/31 (MNRJ) - 25-II-54, WWM; 588, 626/1, 739/46 (FMNH), 747/8 (MZUSP), 749/56 (MNRJ) - 5-XI-54, WWM; 1129 (FMNH), 1152 (MNRJ) - 16-IV-55, WWM. Viamão: 487/8 (FMNH) - 28-III-54, CPJ; (Morro do Côco): 121 (FMNH) - 26-XII-53, WWM.

\section{Hyla minuta Peters, 1872}

RS - Capão da Canoa: 1245 (MNRJ) - 12-III-55, JAP. Farroupilha (18Km S): 989/90 (MNRJ) - 7-II-55, ARC. Gravataí (4Km NE): 1067/9 (MNRJ), 1070/3 (FMNH) - 26-XII-55, WWM \& JAP; (49Km NE): 1060 (MNRJ) 26-XII-55, WWM \& JAP. Osório (Emboabas): 10, 12 (FMNH), 13 (MNRJ) 24-X-53, WWM; 875/84 (FMNH), 885/95 (MNRJ) - 21-XI-54, WWM \& JAP; 1104 (FMNH), 1123 (MNRJ) - 5-III-55, FT. Porto Alegre (Serraria): 1082 (FMNH) - 28-II-55, WWM; 1328 (MNRJ) - 28-III-55, CPJ, EWG, FT \& O. A. 
Pereira; (Teresópolis): 928/33 (FMNHA), 934 (UFRGS), 935/8 (MNRJ) 29-XII-54, WWM. Rio Pardo (Bexiga): 335/9 (FMNH), 343/5 (MNRJ), 623 (UFRGS) - 25-II-54, WWM; 625/8 (MZUSP) - 6-XI-54, WWM, JAP \& ARC.

22. Hyla pulchella Duméril \& Bibron, 1841

RS - Dom Pedrito: 1505/7 (UFRGS) - 1-II-1955, Ac. Osório (Emboabas): 173 (MNRJ) - 8-I-54, WWM; 783 (FMNH), 870 (MNRJ) - 21-XI-54, WWM \& JAP; 1517 (UFRGS) - 25-V-55, JAP. Porto Alegre: 4 (MNRJ) 24-X-53, WWM; 512/3 (MNRJ) - XII-1946, TDL; (Bela Vista): 735/6 (MZUSP), 737/8 (UFRGS) - 9-XI-54, WWM \& JAP. Rio Pardo (Bexiga): 43 (MNRJ), 44/5 (FMNH) - 28-X-53, WWM; 316 MNRJ) - 25-II-54, WWM; 622 (FMNH) - 5-XI-54, WWM, JAP \& ARC; 687/92 (MNRJ), 693/7 (FMNH) 6-XI-54, WWM \& JAP; 1348/9 (FMNH) - 16-IV-55, WWM \& JAP. Torres: 1508 (UFRGS) - 1-XI-54, O. A. Pereira. Viamão (Morro do Côco): 113/6 (FMNH), 117/20 (MNRJ) - 26-XI-53, WWM.

AR - Buenos Aires: Dolores (25Km): 267 (MNRJ) - 5-II-54, WWM \& ARC; 287, 291/3 (FMNH), 288/9 (MNRJ) - 6-II-54, WWM \& ARC.

23. Hyla sanborni Schmidt, 1944

RS - Canoas (Niterói): 552/3 (FMNH) - 29-VII-51, TDL. Capão da Canoa: 1242/3 (FMNH), 1244 (MNRJ) - 12-III-55, JAP. Osório (Emboabas): 14 (MNRJ) - 24-X-55, WWM; 161, 163/6 (MNRJ) - 7-I-54, WWM; 169/72 (MZUSP) - 8-I-54, WWM; 780, 1032/3 (MNRJ), 831/40, 872/4 (FMNH) 20-XI-54, WWM \& JAP; 1110/1 (MNRJ) - 5-III-55, JAP; (2Km SE): 1125 (MNRJ) - 6-III-55, JAP. Rio Pardo (Bexiga): 46 (MNRJ) - 28-XI-53, WWM; 346/9 (MNRJ), 350/2 (FMNH) - 26-II-54, WWM; 629 (FMNH), 685/6 (UFRGS) - 5-XI-54, WWM \& JAP.

24. Hyla squalirostris (Lutz, 1925)

RS - Capão da Canoa (Capão da Canoa): 1245 (MNRJ) - 12-III-55, JAP. Osório (Emboabas): 10, 12 (FMNH), 13 (MNRJ) - 24-X-53, WWM.

Observação. Milstead determinou como "Hyla lindheimeri" (?), nome não encontrado na sinonímia neotropical.

\section{BUFONIDAE}

\section{Bufo Laurenti, 1768}

\section{Bufo arenarum Hensel, 1867}

PR - Ribeirão Claro: 546 (MNRJ) - I-1949, TDL.

RS - Capão da Canoa: 1230/2 (FMNH), 1234/7, 1239/41 (MNRJ) 12-III-55, JAP. São Francisco de Paula: 1351/2 (FMNH) - 26-III-55, EWG. Torres: 986/7 (FMNH) - 4-XI-54, EWG; 1228/9 (MNRJ) - 12-III-55, CPJ; 1387 (MNRJ), 1437/86 (FMNH) - XI-1954, Ac. Tramandaí (Imbé): 63/83 (FMNH), 87, 96,9, 100/6 (MNRJ) - 20-XII-53, WWM \& ARC; (Cidreira): 107/8 MZUSP), 109/10 (UFRGS) - 24-XII-53, CPJ; 1368 (MNRJ) - I-1941, TDL. 
AR - Buenos Aires: Bahia Blanca (20Km W): 194/202 (FMNH) - 2-II-54, WWM \& ARC; 209/19 (FMNH), 220/46 (MNRJ) - 3-II-54, WWM, ARC \& J. I. Townsend.

26. Bufo crucifer Wied, 1821

RS - Osório (10Km SE): 17 (FMNH) - 24-X-53, ARC \& CPJ; 435 (UFRGS) - 5-IV-54, CPJ \& F. M. Salazano.

27. Bufo dorbignyi Duméril \& Bibron, 1841

RS - Osório (Emboabas): 25 (FMNH) - 27-X-53, WWM. Porto Alegre (Ponta Grossa): 573 (MNRJ) - 17-VI-51, TDL; (Teresópolis): 560/3 (FMNH), 564/5 (UFRGS), 566/9 (MNRJ) - 6-VII-1954, TDL. Viamão (Morro do Côco): 703 (MZUSP) - 9-XI-54, WWM \& JAP; 1383 (MNRJ) - 26-XII-53, TDL.

28. Bufo fernandezae Gallardo, 1961

AR - Buenos Aires: Villa G. Conesa: 271 (FMNH) - 6-II-54, WWM \& ARC.

29. Bufo ictericus Spix, 1824

RS - Carlos Barbosa: 1353/4 (MZUSP), 1355 (UFRGS), 1356/61 (FMNH), 1362 (MNRJ) - XI-1.46, TDL.

\section{Melanophryniscus Gallardo, 1961}

30. Melanophryniscus stelzneri dorsalis (Mertens, 1933)

RS - Torres: 27 (MZUSP), 28 (UFRGS), 29 (MNRJ) - 6-XI-53, CPJ 1074 (FMNH) - 18-II-55, S.P. Velasco.

31. Melanophryniscus sp. TDL.

RS - Porto Alegre (Morro de São Pedro): 1512 (FMNH) - 17-IV-55,

\section{PSEUDIDAE}

\section{Pseudis Wagler, 1830}

32.Pseudis minutus Günther, 1858

RS - Capão da Canoa: 1254/8 (MNRJ), 1259/62 (FMNH) - 12-III-55, JAP. Carlos Barbosa: 514/22 (FMNH) - 11-I-46, TDL. Osório (Emboabas): 829 (FMNH), 862/9 (MNRJ) - 20-XI-55, WWM; 1014/27 (FMNH) - 25-II-55, WWM \& JAP; 1096/103 (MNRJ) - 5-III-55, JAP; (16Km SE): 1112 (MNRJ) 5-III-55, JAP. Rio Pardo (Bexiga): 48/50, 53 (MZUSP) - 28-XI-53, WWM; 332/3 (FMNH), 358/62 (MNRJ) - 25-XI-54, WWM; 608/13, 624 (FMNH), 614/19 (MNRJ), 678/84 (UFRGS) - 5-XI-54, WWM, JAP \& ARC; 982 (MNRJ) 14-I-55, WWM; 1132/4 (FMNH), 1135/6 (MNRJ) - 16-IV-55, WWM. Porto Alegre (Serraria): 1083/8 (FMNH), 1089/93, 1277/99, 1300 (MNRJ), 1301/24 (FMNH) - 28-II-55, WWM, CPJ, FT, EWG \& O. A. Pereira; (Teresópolis): 
1164/6 (FMNH) - 9-I-55, TDL \& EWG. Viamão (Morro do Côco): 147/8 $(\mathrm{FMNH})-27-\mathrm{XII}-53$, EWG.

\section{REPTILIA}

SAURIA

IGUANIA

POLYCHRIDAE

I. Anisolepis Boulenger, 1885

1. Anisolepis undulatus (Wiegmann, 1834)

RS - Bento Gonçalves: 1513 (UFRGS) - I-1955, JAP. Carlos Barbosa: 395 (UFRGS) - I-1948, TDL. Farroupilha (18Km S): 429 (FMNH) - 29-III-54, WWM. Garibaldi: 396 (MZUSP) - I-1948, BZL \& TDL. Montenegro (Barão: Vinícola Demarchi): 394 (MNRJ) - 3-II-45, TDL.

\section{TROPIDURIDAE}

\section{Liolaemus Wiegmann, 1834}

2. Liolaemus darwinii (Bell, 1843)

AR - Buenos Aires: Bahia Blanca (17 km W): 247/8 (MZUSP) - 3-II-54; WWM; 253/7 (MNRJ), 258/62 (FMNH) - 4-II-54, WWM.

3. Liolaemus occiptalis Boulenger, 1885

RS - Tramandaí (Cidreira): 390 (MNRJ), 391 (MZUSP) - 20-I-53, W. A. Schmidt \& TDL; 392 (FMNH) - 19-I-52, W. A. Schmidt \& TDL; (Tramandaí): 393 (UFRGS) - 10-VI-51, EWG.

\section{GEKKOMORPHA}

GEKKONOIDEA

GEKKONIDAE

\section{Hemidactylus Gray, 1825}

4. Hemidactylus mabouia (Moreau de Jonnés, 1818) Buckup.

SP - São Paulo (Jardim América): 398 (FMNH) - 20-XII-52, TDL \& L.

RS - Porto Alegre: 1 (MNRJ) - 21-X-53, Ac.; 397 (MZUSP) - XII-1941, TDL; 399 (MNRJ) - 29-I-53, TDL; 400, 402 (FMNH), 401 (MNRJ) - 10-VIII-53 TDL; 424 (UFRGS) - 21-III-54, Ac. 


\section{SCINCOMORPHA}

\section{LACERTOIDEA \\ TEIIDAE}

\section{Cnemidophorus Wagler, 1830}

5. Cnemidophorus lacertoides Duméril \& Bibron, 1839

RS - Osório (Emboabas): 178 (MNRJ), 179 (UFRGS) - 9-I-54, WWM; 785/8 (FMNH), 787 (MNRJ), 788 (MZUSP), 789 (UFRGS) - 21-II-54, WWM; 118/9 (MNRJ), 1120/1 (FMNH) - 6-III-55, WWM.

\section{Pantodactylus Duméril \& Bibron, 1839}

6. Pantodactylus schreibersii schreibersii (Wiegmann, 1834)

RS - Bento Gonçalves: 1514 (UFRGS) - 28-VI-53, JAP. Farroupilha (18Km S): 497 (FMNH) - 4-V-54, WWM. Porto Alegre: 384 (FMNH), 385/6 (UFRGS), 387 (MZUSP), 388 - 1941, TDL \& D. Gomes; 389 (MNRJ) - 1951, L. Buckup \& TDL.

\section{Teius Merrem, 1820}

7. Teius cf. oculatus (d'Orbigny \& Bibron, 1837)

RS - Porto Alegre (Belém Novo): 378/81 (FMNH) - 1941, TDL \& W. A. Schmith; (Ipanema: Morro do Osso): 382 (UFRGS) - 17-VIII-52, E. Pinto; (Teresópolis): 57 (MZUSP) - 9-XII-53, WWM; 61/2 (MNRJ) - 17-XII-53, WWM; 375/7 (MNRJ) - IV-1943, TDL; 485/6 (FMNH) - 22-II-54, WWM; 1226 (FMNH) - 9-I-55, EWG. Viamão (Lomba do Sabão): 383 (UFRGS) - 1-XI-51, TDL \& C. Sottomayor.

Observação: Para CEI \& LESCURE (1985), T. teyou (Daudin, 1802) ocorre para o lado ocidental da Argentina, enquanto que T. oculatus é do NE da Argentina, Uruguai e Brasil.

\section{Tupinambis Daudin, 1803}

8. Tupinambis teguixin (Linnaeus, 1758)

RS - Dom Pedrito: 1488 (Universidade dos E.U.A.), 1489/90 (FMNH), 1941/2 (MNRJ) - 1-II-1955, Ac. Osório (Emboabas): 180 (UFRGS) - 9-I-54, WWM. Porto Alegre: 187 (UFRGS) - 14-I-54, A. Rosa; (Teresópolis): 373 (UFRGS), 374 (MNRJ) - 1951, TDL. Tramandaí (Cidreira): 181 (MNRJ), 182 (FMNH), 183/4 (UFRGS) - 8-I-54, CPJ \& EWG.

Observação: PRESCH (1973) indicou T. rufescens para a parte meridional do continente, e T. teguixin (Linnaeus, 1958) para setentrional. Entretanto, ele viu pouco material e o Dr. José Maria Gallardo (Museo Argentino de Ciências Naturales, Buenos Aires; com. oral) afirma que existem as duas ao norte da Argentina. VANZOLINI (1976) e GUDYNAS (1985) não aceitam o 
estabelecido por PRESCH mas não justificaram seus pontos de vista. Exame do material colecionado na Pontífica Universidade Católica em Porto Alegre, por Marcos Di Bernardo, indica a presença de T. rufecens no RS mas, também, espécimens intermediárias com a outra.

\section{SCINCOIDEA}

\section{SCINCIDAE}

\section{Mabuya Fitzinger, 1826}

9. Mabuya dorsivittata Cope, 1862

SC - Florianópolis (Ilha de SC): 983 (UFRGS) - 29-I-55, FT.

\section{ANGUINOMORPHA}

ANGUOIDEA

ANGUIDAE

\section{Ophiodes Wagler, 1828}

10. Ophiodes striatus (Spix, 1824)

RS - Carlos Barbosa: 411 (FMNH) - I-1947, TDL \& BZL. Porto Alegre: 403 (MZUSP), 404/5 (UFRGS), 406/8 (MNRJ), 409/10 (FMNH) - 1941, TDL.

\section{AMPHISBAENIA}

\section{AMPHISBAENIDAE}

\section{Amphisbaena darwinii trachura Cope, 1885}

RS - Osório (Emboabas): 26 (FMNH) - 27.10.53, WWM. Porto Alegre (Menino Deus): 413 (MZUSP) - 1941, TDL \& W. A. Schmith. Tramandaí: 484 (MNRJ) - 25-III-54, CPJ; 1350 (UFRGS) - 20-II-55, CPJ.

\section{Amphisbaena darwinii ssp.}

RS - Carlos Barbosa: 416 (FMNH) - I-1947, TDL \& BZL. Dom Pedrito: 1504 (UFRGS) - I/II-1955, Ac. Farroupilha (18Km S): 430 (FMNH) - 30-III-54, WWM. Montenegro (Barão): 417/8 (FMNH), 419/20 (MNRJ) - 21-I-46, TDL \& BZL. Nova Petrópolis: 1515 (UFRGS) - III-1955, JAP. Porto Alegre (Bom Fim): 414 (FMNH) - 1940, TDL \& U. Buttgereit; (Serraria): 415 (MNRJ) 9-XII-51, TDL; (Teresópolis): 152 (MZUSP) - VI-1945, TDL \& A. Cardonna. Viamão (Morro do Côco): 150 (FMNH), 151 (MZUSP), 152 (MNRJ) 27-XII-53, TDL \& PF.

Observações: Milstead determinou como A. darwinii, sem indicar subespécie, mas indicando a nominal. GANS (1966) apontou a nominal, para a AR e Uruguai, penetrando ao Sul do Estado onde integrada $\operatorname{com} A$. d. trachura. 
Como o material de Milstead é quase todo do NE do RS, este táxon é outro que não $A$. prunicolor prunicolor (Cope, 1885), que é comum no planalto e de fácil distinção. Há outra espécie descrita, posteriormente, para o Uruguai e que entra neste Estado, A. munoai (Klappenbach, 1960), mas não vimos esta na parte alta do RS.

13. Amphisbaena mertensi Strauch, 1881

PR - Ribeirão Claro: 421 (FMNH), 422 (MNRJ) - I-1949, TDL.

$$
\text { XI. Anops Bell, } 1833
$$

14. Anops kingii Bell, 1833

RS - Porto Alegre (Floresta): 423(FMNH)-24-V-51, TDL \& D. Buriak.

\section{SERPENTES}

\section{COLUBROIDEA \\ COLUBRIDAE}

Observação: Foram omitidos os nomes das subfamílias porque estão em análise por diferentes AA cujas propostas são, ainda, objeto de discussão porque ainda faltam muitos dados dos táxons sulamericanos.

\section{Atractus Wagler, 1828}

15. Atractus reticulatus ssp.

RS - Porto Alegre (Tristeza): 506 (UFRGS) - 16-VI-54, Ac.

Observações: PETERS \& OREJAS-MIRANDA (1970) indicam a nominal para o Brasil, mas vimos que há outras formas envolvidas (em estudo).

O exemplar foi visto enterrado em solo de terra frouxa.

\section{Chironius Fitzinger, 1826}

16. Chironius bicarinatus (Wied, 1820)

RS - Caxias do Sul (Ana Rech): 1342 (FMNH) - 8-IV-55, EWG.

\section{Clelia Fitzinger, 1826}

17. Clelia occipitolutea (Duméril, Bibron \& Duméril, 1854)

RS - Farroupilha (32Km S): 490 (FMNH) - 3-V-54, WWM. Rio Pardo (52Km E): 960 (UFRGS) - 12-I-55, WWM.

Observações: O 490 foi visto D.O.R., em tarde quente, céu coberto. O 960, também D.O.R., meio da manhã; antes de fixado mostrava dorsalmente um azul quase anil (irisado), empalidescendo para cor de vinho nos lados, sendo branco-rosado o ventre (Milstead diz que a cor dorsal é vermelha-azulada). 


\section{Elapomorphus Wiegmann, 1843}

\section{XVa. Elapomorphus (Phalotris) Cope, 1862}

18. Elapomorphus $(P$.) lemniscatus trilineatus Boulenger, 1889

RS - Osório (Emboabas): 452 (UFRGS) - 6-IV-54, WWM; 1518 (FMNH) - 25-V-55, JAP.

Observações: $\mathrm{O}$ exemplar 452 estava em montículo de areia na entrada de uma galeria de Ctenomys sp. (Mammalia, Rodentia), em zona de campo, de manhã. Ostentava cor dorsal mimética com a areia.

\section{Helicops Wagler, 1830}

\section{Helicops infrataeniatus (Jan, 1865)}

RS - Guaíba (44Km W): 312 (MNRJ) - 25-II-54, WWM \& CPJ. Osório (8Km SE): 479 (FMNH) - 6-IV-54, WWM \& CPJ. Porto Alegre (Belém Novo): 1346 (MNRJ) - 12-IV-55, EWG; (Tristeza, Rio Guaíba): 153 (UFRGS) - 1-I-54, C. Machado. S. Leopoldo (Rio dos Sinos): 188 (FMNH) - 17-I-54, WWM.

Observações: O nome usado segue DEIQUES \& CECHIN (1991). O exemplar 153 estava na areia da praia, dia de sol; o 188 nadava no rio, próximo a uma escadaria de praça no centro da cidade; o 312 foi visto D.O.R., após duas horas de fortes chuvas, estando toda a região alagada, inclusive os lados da rodovia, em valas; o 479, D.O.R., justo no ponto em que um arroio tubulado cruza a rodovia, no início de tarde.

\section{Liophis Wagler, 1830}

20. Liophis almadensis (Wagler, 1824)

RS - Gravataí (12Km E): 481 (MNRJ) - 6-IV-54, WWM \& CPJ. Osório (Emboabas): 1122 (FMNH) - 6-III-55, JAP \& FT. Rio Pardo (Bexiga): 701 (UFRGS) - 6-XI-54, WWM. Santo Antônio da Patrulha (30Km SW): 1094 (FMNH) - 5-III-55, WWM. Viamão: 1227 (MNRJ) - III-1955, CPJ.

Observações: O exemplar 481 foi visto D.O.R., início da tarde; 701 estava sob o tronco, savana aberta, meio da tarde; o 1094, D.O.R., meio da tarde; o 1122 estava dentro do arroio, entre folhas de uma associação de filodêndros, às 11:30hs, dia de sol brilhante, ar fresco.

\section{Liophis anomalus (Günther, 1858)}

RS - Porto Alegre (Belém Novo, 11KM SE): 1267 (FMNH) - 30-III-55, WWM \& J. L. Milstead. Rio Pardo (34Km E): 582 (UFRGS) - 5-XI-54, WWM.

AR - Buenos Aires: Azul (135Km NE): 192 (FMNH) - 28-I-54, WWM \& ARC; (92Km SSW): 193 (MNRJ) - 29-I-54, WWM \& ARC; Dolores: 263/4 (FMNH), 265/6 (MNRJ) - 5-II-54, WWM \& ARC. 

\& ARC.

AR - Entre Rios: Paraná (62Km NE): 306 (FMNH) - 13-II-54, WWM

Observações: O 192 foi visto D.O.R., final da tarde. O 193 D.O.R., na rodovia para Bahía Blanca, pela manhã. Os 263/6, D.O.R., final da tarde; estavam entre outros que não foram colhidos por estarem despedaçados, distando cerca de 4 e $6 \mathrm{Km}$ um do outro; região plana, costeira, alagada por chuvas, havendo grandes valas cheias de água nos lados da rodovia. O 306 foi encontrado D.O.R., no meio da manhã. O 582, D.O.R., ao final da manhã.

\section{Liophis flavifrenatus (Cope, 1862)}

RS - Rio Pardo (50Km E): 961 (UFRGS) - 12-I-55, WWM.

Observações: Encontrado D.O.R., meio da manhã. Antes de ser fixada, apresentava a cor de fundo castanha clara, rosada, com uma estria vermelha vertebral, uma estria amarela lateral, ambas convergindo no rostral; uma estria preta lateral sobre a II e III filas de dorsais; ventre branco amarelado com manchas pretas semilunares cruzadas longitudinalmente por linha rosa-pálido, não visível em toda a extensão.

23. Liophis jaegeri jaegeri (Günther, 1858)

RS - Rio Pardo (26Km E): 585 (FMNH) - 5-XI-54, WWM. Viamão (Morro do Côco): 149 (UFRGS) - 27-XII-53, EWG.

Observações: O 149 foi encontrado sob rocha, campo ao lado do Rio Guaíba, pela manhã, dia parcialmente nublado, com sol fraco. O 585 foi visto D.O.R., ao final da tarde; antes de fixado apresentava a coloração verde esmeralda no dorso, com uma estria vertebral de cor ferrúginea, e o ventre vermelho coral.

\section{Liophis miliaris ssp.}

SC - Florianópolis (Ilha de Santa Catarina): 984 (MNRJ) - 28-I-55, FT. WWM.

RS - São Francisco de Paula (9Km SW): 897 (UFRGS) - 14-XI-54,

AR - Santa Fé: Maciel (10Km S): 190 (FMNH) - 25-I-54, WWM \& ARC.

Observações: O 190 foi encontrado D.O.R., final da tarde. O 867, D.O.R., meio da manhã, região elevada. O 984 foi visto na praia, andando na areia.

\section{Liophis poecilogyrus ssp.}

RS - Dom Pedrito: 1502 (FMNH), 1503 (UFRGS) - I/II-1955, Ac. Farroupilha (41Km S): 498 (MNRJ) - 4-V-54, WWM. Gravataí (13Km E): 18 (MNRJ) - 27-X-54, WWM \& ARC; (16Km E): 19 (FMNH) - 27-X-54, WWM \& ARC. Guaíba (14Km W): 957 (MNRJ) - 12-I-55, WWM. Porto Alegre (Belém Novo: 8Km SE): 1264 (FMNH) -30-III-55, WWM \& J. L. Milstead. Rio Pardo (Bexiga): 371 (FMNH) - 25-V-54, WWM; 702 (UFRGS) - 6-XI-54, WWM; (128Km E): 579 (FMNH) - 5-I-54, WWM. Santo Antônio da Patrulha: 20 (MNRJ) - 27-X-54, WWM \& ARC. Torres (Lagoa Itapeva): 33 (FMNH) 6-XI-53, CPJ. Tramandaí (Cidreira: Lagoa do Lessa): 186 (MNRJ) - 8-I-54, 
WWM \& EWG. Viamão: 1227 (MNRJ) - III-1955, CPJ.

Observações: DIXON (1980) não considera as subespécies de AMARAL (1944) e afirma haver um mosaico na distribuição geográfica das mesmas (com. epist.). Entretanto, dois exemplares da coleção Milstead estão identificados a nível subespecífico e os apresentados adiante. AMARAL (1944) aponta para o lado oriental do RS e SC, L. poecilogyrus pictostriatus, mas um exame preliminar da variação mostrou certa complexidade que exige uma análise populacional, inclusive com variação ontogênica somada.

O 1820 foi encontrado D.O.R., início da manhã em dia com fortes chuvas. O 93, D.O.R., próximo a lagoa. O 371 estava dentro de tronco podre tombado, em campo plano, final de tarde. O 498, D.O.R., meio da tarde com sol brilhante, temperatura alta. O 579, D.O.R., início da manhã. O 702 estava dentro de tronco podre tombado dentro de mata, meio da tarde. O 957, D.O.R., início da manhã.

26. Liophis poecilogynus platensis (Amaral, 1944)

AR - Buenos Aires: Magdalena (20Km SE): 300 (FMNH) - 11-II-54, WWM \& ARC.

Observação: Estava A.O.R., cruzando o bosque cultivado de coníferas, final da tarde, na fazenda Pearson.

27. Liophis poecilogyrus subfasciatus Cope, 1862

RS - São Vicente do Sul (General Vargas: 36Km E): 308 (FMNH) 14-II-54, WWM.

Observação: Vista A.O.R., próximo rio Jaguarão, início da manhã.

\section{Lystrophis Cope, 1885}

28. Lystrophis dorbignyi ssp.

RS - Dom Pedrito: 1493/6 (FMNH), $1497 / 9$ (MNRJ) - I/II-1955, Ac. Porto Alegre (Belém Novo: 9Km SE): 1265 (UFRGS) - 30-III-55, WWM \& J. L. Milstead. Rio Pardo (54Km E): 581 (FMNH) - 5-XI-54, WWM; (79Km E): 700 (MNRJ) - 5-XI-54, WWM. Santo Antônio da Patrulha (19Km E): 480 (MNRJ) - 6-IV-54, WWM \& CPJ. Tramandaí (Cidreira: Lagoa do Lessa): 185 (UFRGS) - 8-I-54, CPJ \& EWG.

AR - Entre Rios: Paraná (20Km NE): 304 (MNRJ) - 13-II-54, WWM \& ARC; (90Km NE): 307 (FMNH) - 13-II-54, WWM \& ARC.

Observações: Milstead notou a grande variação que apresenta esta espécie e, do diálogo conosco, nasceu um trabalho que está em andamento cujos resultados prévios foram comunicados (LEMA \& ARAUJO, 1983).

O 185 estava andando em campo, próximo a uma pilha de troncos, à tarde, região de solo arenoso. O 304 e 581 estavam D.O.R., meio da manhã. O 307, D.O.R., de manhã. O 480, D.O.R., meio da tarde. O 700, A.O.R., início da manhã. O 1265, A.O.R., ao fim da tarde.

O 185 tem a coloração e contagem de escamas semelhante aos 305 e 480, mas difere por ter o IV supralabial tocando órbita, carácter que, na chave de 
BOULENGER (1886), identifica L. histricus (Jan, 1863) mas, HOGE et al. (1976), dizem ser próprio de L. dorbignyi.

\section{Mastigodryas Amaral, 1935}

29. Mastigodryas bifossatus ssp.

RS - Capão da Canoa: 1270 (FMNH) -2-IV-55, WWM \& J. L. Milstead.

XX. Oxyrhopus Wagler, 1830

30. Oxyrhopus rhombifer rhombifer Duméril, Bibron \& Duméril, 1854

RS - Porto Alegre: 482 (UFRGS) - II-1954, Ac.

\section{Philodryas Wagler, 1830}

31. Philodryas aestivus subcarinatus Boulenger, 1902

RS - Osório (19Km NE): 503 (UFRGS) - 20-V-54, WWM. Torres (Lagoa Itapeva): 32 (FMNH) - 6-XI-53, CPJ.

Observações: $\mathrm{O} 32$ foi capturado próximo às margens da lagoa, em pequeno capão. O 503, A.O.R., no meio da tarde, dia de sol brilhante, mas fresco.

32. Philodryas olfersii olfersii (Lichtenstein, 1823)

RS - Rio Pardo (Bexiga): 42 (MNRJ) - 28-XI-53, WWM; 587 (UFRGS) - 5-XI-54, JAP \& ARC; (30Km W): 962 (FMNH) - 12-I-55, WWM. Viamão (Morro do Côco): 111 (FMNH) - 26-XII-53, TDL.

Observações: O 42 estava dormindo dentro das folhas de bromélia fastuosa, no lado SW de mata, dia frio, manhã, sol fraco, parcialmente nublado. O 111 cruzava trilha de mata. Cerca das $10 \mathrm{hs}$, manhã, com sol brilhante mas o local sombrio, de vegetação densa; após captura regurgitou os membros posteriores de Hyla sp., pequena. O 587 estava andando no chão do mato, no meio da tarde; viva apresentava coloração dorsal verde esmeralda, com estria vertebral ferrugínea até o topo da cabeça que apresenta faixa preta lateral, da narina à tempora sobre olho, atingindo atrás do ângulo bucal, onde afina e enfraquece; face ventral cefálica verde esmeralda, mento e ventre do corpo verde-azulados. O 962, D.O.R., início da tarde, coloração similar à anterior (587). O 42, viva, apresentava coloração verde brilhante com estria ferrugínea vertebral atingindo sobre a cabeça, lateralmente verde, estria post-ocular atingindo o ângulo oral, e assim, também o 111.

\section{Philodryas patagoniensis ssp.}

RS - Guaíba (19Km W): 958 (MNRJ) - 12-I-55, WWM. Porto Alegre (Itapuã: 8Km NW): 1269 (MNRJ) - 30-III-55, WWM \& J. L. Milstead; (Petrópolis): 985 (FMNH) - 10-I-54, EWG. Rio Pardo (44Km E): 580 (UFRGS) - 
5-XI-54, WWM; (26Km E): 583/4 (MNRJ) - 5-XI-54, WWM; (21Km E): 586 (FMNH) - 5-XI-54, WWM, São Francisco de Paula (2 km NE): 899 (FMNH) - 14-XI-54, WWM; (11Km NE): 898 (FMNH) - 14-XI-54, WWM; (30Km NE): 902 (FMNH) - 14-XI-54, WWM. São Leopoldo (5Km N): 1263 (FMNH) 21-III-55, WWM. Viamão (Tarumã): 988 (MNRJ) - 20-II-55, I. D. Pinto.

AR - Buenos Aires: Bahía Blanca (19Km W): 203 (FMNH) - 3-II-54, WWM \& ARC.

Observações: O 203 foi visto D.O.R., início da manhã, região alagada devido fortes chuvas, com valas cheias de água aos lados da rodovia. O 580 foi visto D.O.R., no início da manhã, também em região alagada por chuvas. Exemplares 583/6 e 898, todos D.O.R., final de manhã. O 899 também D.O.R., final da tarde. O 902, A.O.R., meio da tarde. O 958, D.O.R., início da manhã. O 988, D.O.R. O 1263, D.O.R., pela tarde. O 1269, D.O.R., final de tarde.

\section{Rhadinaea Cope, 1863}

\section{Rhadinaea occipitalis (Jan, 1863)}

RS - Porto Alegre (Belém Novo: 11Km SE): 1268 (UFRGS) - 30-III-55, WWM \& J. L. Milstead.

Observação: Milstead identificou-a como "Leimadophis melanostigma" Wagler, 1824, mas esta espécie possui 17 filas de dorsais sem redução, 24 a 33 dentes maxilares e três labiais em contato com órbita. Outrossim, $L$. melanostigma está sendo partida em outros táxons, e assim também as espécies do grupo brevirostris do gênero Rhadinaea que deverão ser alocados em gênero novo. (Em estudo).

\section{Rhabdinaea poecilopogon Cope, 1863}

RS - Capão da Canoa: 1487 (FMNH) - 28-III-55, JAP.

\section{Sibynomorphus Fitzinger, 1843}

36. Sibynomorphus ventrimaculatus (Boulenger, 1885)

RS - Porto Alegre (Morro da Polícia): 483 (MNRJ) - 25-III-54, Ac.; (Tristeza): 954/5 (FMNH), 956 (MNRJ) - 12-I-55, WWM. Rio Pardo (Bexiga): 372 (UFRGS) - 26-II-54, WWM.

Observações: $\mathrm{O} 372$ foi coletado no topo de um arbusto no ângulo de um campo alagado, á noite; quando segura ativou-se. Os 954/6 foram vistos D.O.R., início da manhã.

XXIV. Tomodon Duméril \& Bibron, 1853

37. Tomodon dorsatus Duméril, Bibron \& Duméril, 1854

RS - Viamão (Morro do Côco): 112 (UFRGS) - 26-XII-53, WWM. 
Observações: "Andando" no chão de mata cerca das 17:00hs, com sol brilhante mas sombrio no local do encontro.

38. Tomodon ocellatus Duméril, Bibron \& Duméril, 1854

AR - Buenos Aires: Pergamino (121Km SE): 191 (UFRGS) - 26-I-54, WWM \& ARC.

AR - Entre Ríos: Paraná (17Km NE): 301/2 (MNRJ), 303 (FMNH) 13-II-54, WWM \& ARC; (58Km NE): 305 (FMNH) - 13-II-54, WWM \& ARC.

Observações: O 191 estava D.O.R. para Buenos Aires, meio da manhã. Os 301/3 e 305 estavam D.O.R., no meio da manhã.

\section{Waglerophis Romano \& Hoge, 1973}

39. Waglerophis merremii (Wagler, 1824)

RS - Osório (Emboabas): 56 (UFRGS) - 24-X-53, WWM. Porto Alegre (Belém Novo: 10Km SE): 1266 (FMNH) - 30-III-55, WWM \& J. L. Milstead.

Observações: O 56 estava ativo no ângulo da floresta, às $15 \mathrm{hs} \mathrm{de} \mathrm{dia} \mathrm{frio;}$ quando alguém se aproximava dela, achatava-se contra o solo; mantida em laboratório por vários dias, alimentou-se de Leptodactylus ocellatus. O 1266 foi visto D.O.R., ao final de tarde.

\section{ELAPIDAE}

\section{MICRURINAE}

\section{Micrurus Wagler, 1824}

40. Micrurus corallinus (Merrem, 1820)

RS - Dom Pedrito: 1500 (FMNH), 1501 (UFRGS) - I/II-1955, Ac.

Observação: A ocorrência na Serra de Sudeste parece errada mas, considerando que muitos representantes da fauna do planalto meridional fixam-se naquela área elevada (LEMA, 1982), bem como já ter sido encontrada na área, é possível aceitar a ocorrência.

\section{Micrurus frontalis ssp.}

RS - Guaíba (63Km W): 959 (FMNH) - 12-I-55, WWM. Porto Alegre: 489 (UFRGS) - 29-IV-54, Ac.

Observações: $\mathrm{Na}$ área predomina $M$. frontalis altirostris (Cope, 1860), mas a NE do RS surgem tipos integradantes ou de outro táxon, alguns, por sua alta contagem de tríades, enquadram-se em M. frontalis multicinctus Amaral, 1944 e que HOGE \& ROMANO-HOGE (1978/79) consideraram como produto da intergradação da nominal com a pampeana (citada antes).

O 959 foi visto D.O.R., meio da manhã. 


\section{VIPERIDAE}

\section{CROTALINAE}

\section{Crotalus Linnaeus, 1758}

\section{Crotalus durissus terrificus (Laurenti, 1768)} Paulo.

SP - São Paulo?: 504/5 (UFRGS) - 25-VIII-53, Universidade de São

Observação: Ambas foram vistas cruzando a rua no campus da USP, onde há um pequeno horto que é uma reserva da biota da área. Mas é possível que tenham fugido dos viveiros do Instituto Butantan, anexo ao campus.

\section{REFERÊNCIAS BIBLIOGRÁFICAS}

AMARAL, A. DO. 1944. Nota sobre a Ofiologia neotrópica e brasílica. 1. Distribuição geográfica e racial de Leimadophis poecilogyrus (Wied). Papéis Av. Depto Zool. S. Paulo 5 (10): 75-82.

BOULENGER, G.A. 1886. A synopsis of the reptiles and batrachians of the Province Rio Grande do Sul, Brazil. Ann. Mag. nat. Hist. 18: 423-45.

CEI, J.M. \& J. LESCURE. 1985. Identité de Teius teyou (Daudin, 1802), type du genre et de $T$. oculatus (D'Oebigny et Bibron, 1837) (Reptília, Teiídae). Bull. Mus. natn. Hist. nat., Paris (4e. sér) 7 (séct. A) (2): 461-73.

DEIQUES, C.H. \& S.T.Z. CECHIN. 1991. O status de Helicops carinicaudus (Wied, 1825). (Serpentes: Colubridae). Acta biol. leopoldensia (1990) 12 (2): 313-26.

DIXON, J.R. 1980. The neotropical colubrid snake genus Liophis. The generic concept. Contr. Biol. Geol. Milwaukee Mus. (31): 1-40.

DOWLING, H.G. \& W.E. DUELLMAN. 1978. Systematic Herpetology. New York, HISS Publ. (Amer. Mus. Nat. Hist.).

ESTES R. \& G. PREGILL. 1988. Phylogenetic Relationships of the Lizards Families. Essays commemorating Chales L. Camp. Stanford, Stanford Univ. FROST, D.R. \& R. ETHERIDGE. 1989. A phylogenetic analysis and taxonomy of iguanian lizards (Reptilia: Squamata). Misc. Publ. Mus. Nat. Hist. Univ. Kansas 81: 1-65.

GANS, C. 1966. Studies on amphisbaenids (Amphisbaenia, Reptilia). 3. The small species from southern South America commonly identified as Amphisbaena darwini. Bull. Amer. Mus. Nat. Hist., 134:185-260.

GUDYNAS, E. 1985. Notas sobre teiidos del Uruguai. (Lacertilia Teiidae). 1. Nuevos registros y distribución geográfica de Tupinambis tequixin, etc. Contrib. Biol. Centro Educ. D. Orione, Montevideo, 12:9-17.

HOGE, A.R.; C.L.S. CORDEIRO \& S.A.L. ROMANO. 1976. Posição taxonômica de Lystrophis nattereri (Steindachner) (Serpentes, Colubridae). Mem. Inst. Butantan (1975) 39:37-50.

HOGE, A.R. \& S.A.R.W.L. ROMANO-HOGE. 1978/79. Sinopse das espécies 
peçonhentas do Brasil ( $2^{\mathrm{a}}$ ed.). Mem. Inst. Butantan 42/43: 373-496.

KLAPPENBACH, M.A. 1960. Notas Herpetológicas. 1. Amphisbaena munoai n.sp. (Amphisbaenidae). Comun. Zool. Mus. Hist. Nat. Montevideo, 4 (84): $1-12$.

LAURENT, R.F. 1967. Taxonomia de los anuros. Acta zool. lilloana 22: 207-9. LEMA, T. DE. 1982. Fauna de serpentes da província Pampeana e interrelações com as províncias limítrofes. Mem. Inst. Butantan, 46: 173-82.

LEMA, T. DE \& M.L. DE ARAUJO. 1983. Análise da variação de Lystrophis dorbignyi (Duméril, Bobron et Duméril, 1854). Nota prévia. (Serpentes, Colubridae, Heterodontinae). Resumos X Congresso Brasileiro de Zoologia, p.321-322.

MILSTEAD, W.W. 1956. A small colletion of amphibians and reptiles from Argentina. Rev. Bras. Biol. 16 (3): 321-325.

- 1961. Notes on teid lizards in southern Brazil. Copeia (1961) 4: 493-495.

PETERS, J.A. \& B. OREJAS-MIRANDA. 1970. Catalogue of the Neotropical Squamata. Part. 1, Snakes. Bull U.S. Nat. Mus. 297: 1-347.

PRESH, W. 1973. A review of the tegus lizard genus Tupinambis (Sauria: Teiidae) from South America. Copeia (1973) 4: 740-6.

TAYLOR, E.H. 1968. Caecillians of the world. Lawrence, Univ. Kansas, 583p. VANZOLINI, P.E. 1976. On the lizards of a cerrado-caatinga contacts: evolutionary and zoogeographical implications (Sauria). Papeis Av. Depto Zool. S. Paulo 29 (16): 111-9.

Recebido em 15.VII.1991; aceito em 25.XI.1993. 